This item was submitted to Loughborough's Research Repository by the author.

Items in Figshare are protected by copyright, with all rights reserved, unless otherwise indicated.

\title{
Effect of hip flexibility on optimal stalder performances on high bar
}

PLEASE CITE THE PUBLISHED VERSION

PUBLISHER

(C) Taylor \& Francis

VERSION

AM (Accepted Manuscript)

LICENCE

CC BY-NC-ND 4.0

REPOSITORY RECORD

Begon, Mickael, Michael J. Hiley, and Maurice R. Yeadon. 2019. "Effect of Hip Flexibility on Optimal Stalder Performances on High Bar”. figshare. https://hdl.handle.net/2134/5916. 
This item was submitted to Loughborough's Institutional Repository (https://dspace.lboro.ac.uk/) by the author and is made available under the following Creative Commons Licence conditions.

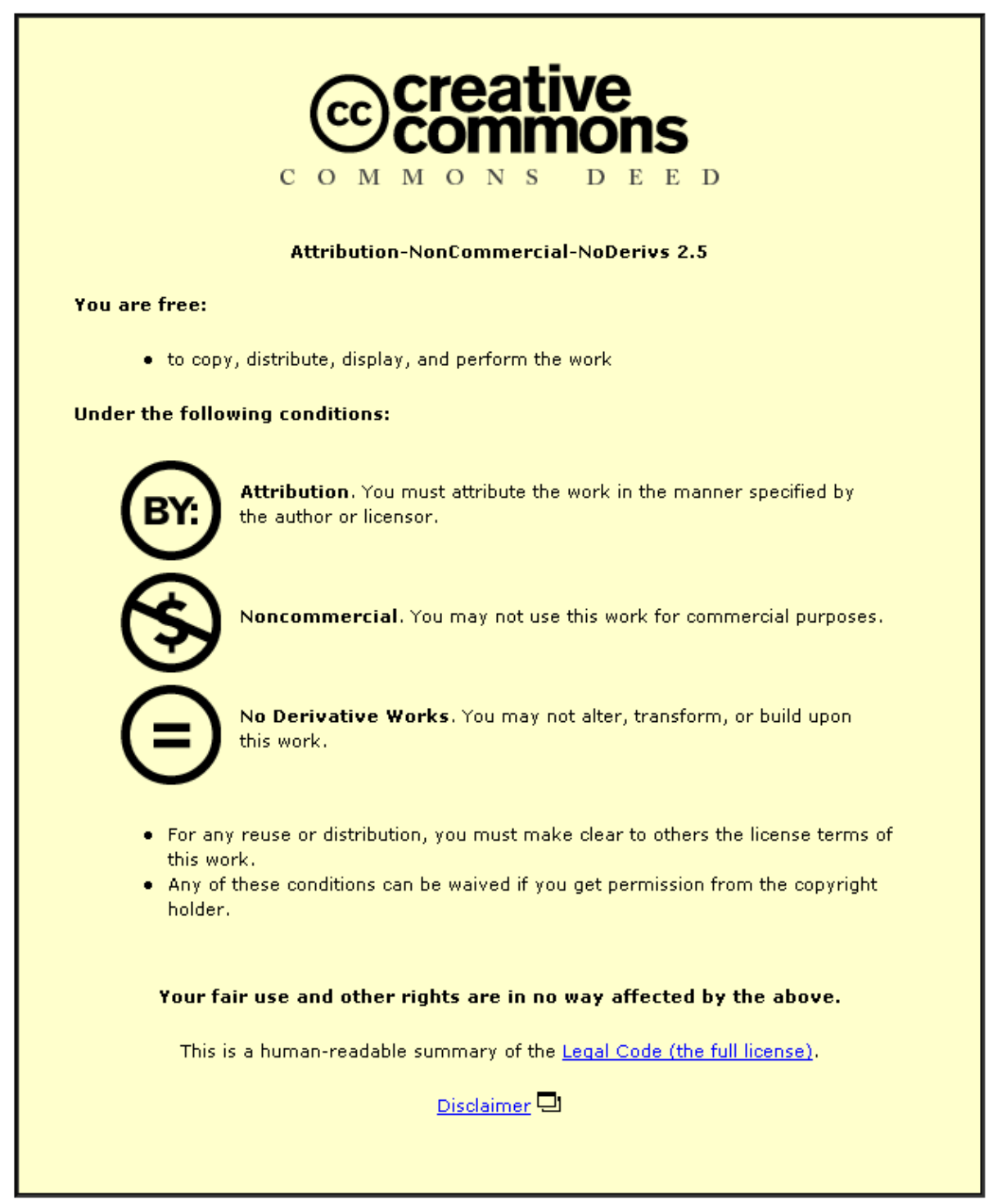

For the full text of this licence, please go to: http://creativecommons.org/licenses/by-nc-nd/2.5/ 
Computer Methods in Biomechanics and Biomedical Engineering 2009, 12 (5), 575-583.

\title{
RESEARCH NOTE
}

\section{Effect of hip flexibility on optimal stalder performances on high bar.}

\author{
Mickaël Begon $^{\mathrm{ab}^{*}}$, Michael J. Hiley ${ }^{\mathrm{c}}$ and Maurice R. Yeadon ${ }^{\mathrm{c}}$ \\ ${ }^{a}$ Department of Kinesiology, University of Montreal, Montreal (Qc), Canada, ${ }^{b}$ Sainte-Justine \\ Hospital Research Centre, Montreal (Qc), Canada; \\ ${ }^{c}$ School of Sport and Exercise Sciences, Loughborough University, Loughborough, United \\ Kingdom
}

*Corresponding author. Email: mickael.begon@umontreal.ca

\begin{abstract}
In the optimization of sports movements using computer simulation models, the joint actuators must be constrained in order to obtain realistic results. In models of a gymnast, the main constraint used in previous studies was maximum voluntary active joint torque. In the stalder, gymnasts reach their maximal hip flexion under the bar. The purpose of this study was to introduce a model of passive torque to assess the effect of the gymnast's flexibility on the technique of the straddled stalder. A threedimensional kinematics driven simulation model was developed. The kinematics of the shoulder flexion, hip flexion and hip abduction were optimized to minimize torques for four hip flexion flexibilities: $100^{\circ}$, $110^{\circ}, 120^{\circ}$ and $130^{\circ}$. With decreased flexibility, the piked posture period is shorter and occurs later. Moreover the peaks of shoulder and hip torques increase. Gymnasts with low hip flexibility need to be stronger to achieve a stalder; hip flexibility should be considered by coaches before teaching this skill.
\end{abstract}

Keywords: simulation, gymnastics, coordination, passive torque

\section{Introduction}

A high bar routine in men's artistic gymnastics consists of a number of circling skills, release and regrasp skills, and a dismount. Previous biomechanical analyses have focused on the transfer of energy between the gymnast and the bar (Arampatzis \& Brüggemann, 1998; 2001) or on the technique of the giant circles on high bar and asymmetrical bars based on a six degrees of freedom (DoF) planar computer simulation model (Hiley \& Yeadon, 2003ab; 2005ab; 2007). The studies investigated the ability to generate angular momentum (Hiley \& Yeadon, 2003a; 2005a), the margin of error for dismounts (Hiley \& Yeadon, 2003b; 2005b) or the consistency of release and regrasp skills (Hiley et al., 2007).

Among the circling skills there are elements in which the gymnast moves close to the bar such as endo (free circle forward) and stalder (free circle backward, Figure 1). The gymnast swings to handstand with a piked posture as he passes through the lower part of the circle. The basic technique of the stalder (Figure 1) comprises a "closing phase" (b-c) reaching the fully piked posture (d-e) with legs between the bar and the shoulders. The fully closed posture may either be piked (legs together) or straddled (legs apart) and is held for a period before extending to handstand: "opening phase" (f). 


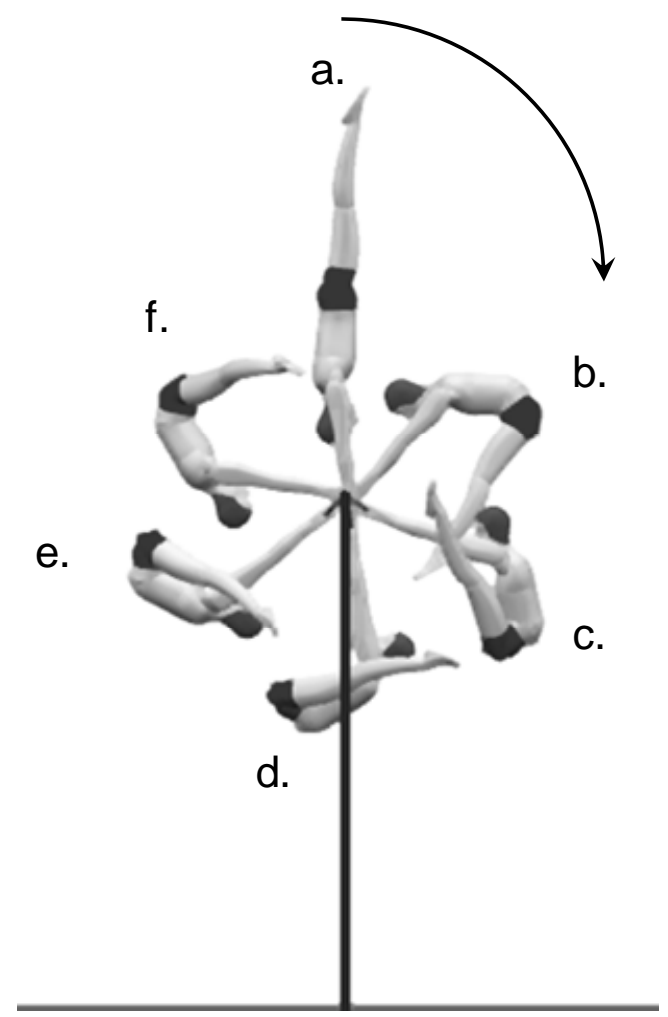

Figure 1: Stalder from handstand to handstand.

Due to changes in the FIG (Fédération Internationale Gymnastique) Code of Points (FIG, 2006), these skills have recently increased in popularity with additional difficulty points available for adding longitudinal turns during the opening phase. However the element must always be completed in a nominal handstand (within $\pm 15^{\circ}$ of the vertical). Such elements can be linked by giant circles or linked directly together without additional swings. Inter-segmental coordination and flexibility seem to be the main qualities required to perform this movement (Pedescu, 1985). There exist various techniques according to the physical qualities of the gymnast.

Due to gravity and centrifugal effects, the gymnast reaches maximal hip flexion under the bar. The stretching of soft tissue (muscles, tendons and ligaments) crossing the hip joint generates passive joint moments $-\tau_{\mathrm{P}}-$ that are not negligible (Hoang et al., 2005). This component of joint torque has not been introduced in previous models of high bar simulation. Since the giant circle performance depends mainly on coordination and the ability to produce large joint torques, complex models of maximum voluntary active joint torque $-\tau_{\mathrm{A}}$ - have been developed (Yeadon et al., 2006) and then introduced into optimization procedures to obtain realistic movements.

In the case of movements reaching the joint end-range of motion, the utilization of a passive torque model is essential. Exponential models of passive moment-angle relationships (Yoon and Mansour, 1982; Davy and Audu, 1987; Hoang et al., 2005) have previously been used to capture the sharp increase in force that occurs near the end-range of motion. This paper investigates the optimization of straddled stalders from handstand to handstand for different gymnast flexibilities. The purpose is to determine the change in coordination with decreasing hip flexibility. 


\section{Methods}

A combination of experimental and theoretical approaches was used. Video data were collected on an elite gymnast performing straddled stalders from handstand to handstand. The kinematics was reconstructed using a chain model presented in Begon et al. (2008) combined with an optimization procedure. Based on this chain model, the computer simulation model was simplified by introducing damped springs and coupling motions; the shoulder translation is expressed as a function of its flexion and the back flexion depends on the hip flexion and abduction. Finally, the simulation model was used to optimize the performance in the stalder for four different hip flexibilities based on the integral quadratic amount of active torques.

\section{Data collection and analysis}

The participant, a member of the Great Britain Men's Senior Gymnastics Squad (17 years old, $61.6 \mathrm{~kg}, 1.705 \mathrm{~m}$ ), gave informed consent to perform setup movements (to estimate the parameters of a personalized chain model) and also a number of stalders varying in technique and speed from trial to trial according to specific instructions. The gymnast was linked to the bar using loops and gloves to minimize the friction with the bar. A set of 95 anthropometric measurements were taken on the gymnast and inertia parameters were estimated using the model of Yeadon (1990). Five successful stalders (i.e. to handstand) were selected to give a range of technique (Figure 2).
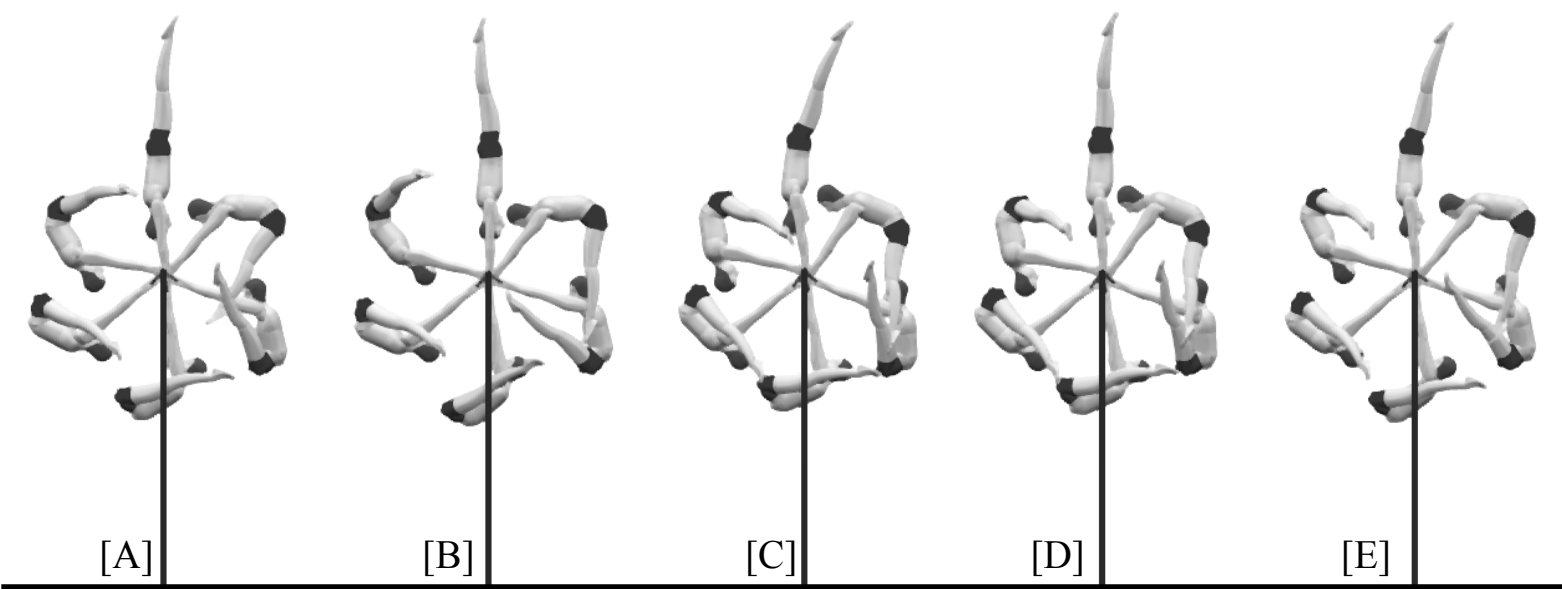

Figure 2: Graphical sequences of the five actual stalders from handstand to handstand with specific instructions: [A] self-selected technique, [B] pike later, [C] as slow as possible, [D] start with shoulder flexion, [E] as fast as possible.

All trials were captured using an automatic 18 camera system (VICON Motion Capture Systems, Oxford, England) operating at $100 \mathrm{~Hz}$ in a $3 \times 5 \times 5 \mathrm{~m}$ calibrated volume centred on the high bar. A six-parameter, three-dimensional, 12 DoF model was used to describe the kinematics of the straddled stalder (Begon et al., 2008; Figure 3A). Ten $25 \mathrm{~mm}$ diameter spherical markers were attached to the left side of the body at the lateral malleolus, tibia, lateral knee, lateral side of mid-thigh, left anterosuperior and posterosuperior iliac spines, xiphoid, first thoracic vertebra, olecranon, ulnar styloid plus two markers to locate the middle of the bar. Markers were added to estimate the location of left ankle, knee and wrist joint centres (medial malleolus, medial femoral condyle and radial styloid) as midpoints and to functionally determine the left hip and shoulder joint centres (right anterosuperior and posterosuperior iliac spines, manubrium, a rigid tripod fixed 
on the left acromion, under the left deltoid and medial side of the left elbow) using the SCoRE algorithm (Ehrig et al., 2008). The model was considered to be symmetrical about the sagittal plane. The 12 generalised coordinates were optimized for each frame by means of a Newton-Gauss nonlinear least squares algorithm under the HuMAnS toolbox (Wieber et al., 2006).

[A] Data collection

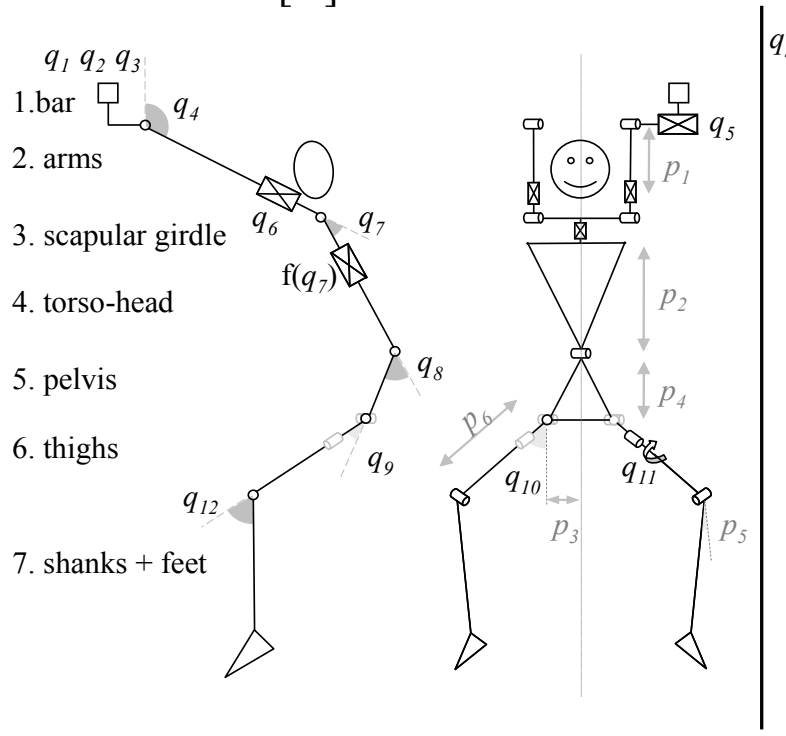

[B] Simulation

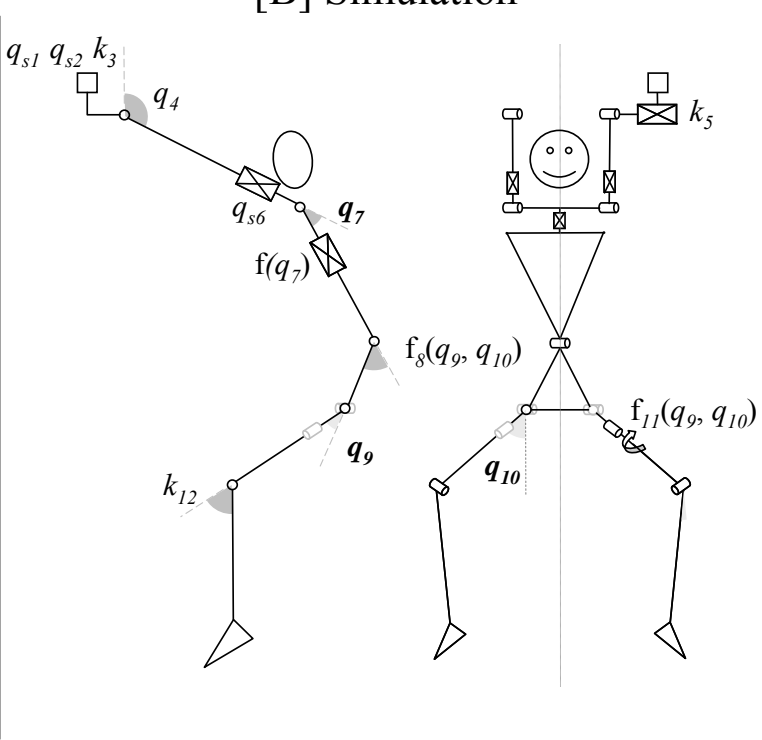

Figure 3: [A] Model definition with the degrees of freedom and the parameters. Degrees of freedom: $q_{1-3}$ translation of the bar, $q_{4}$ arm rotation, $q_{5}$ body translation, $q_{6}$ arm lengthening, $q_{7}$ shoulder flexion, $q_{8}$ spinal flexion, $q_{9}$ thigh flexion, $q_{10}$ thigh abduction, $q_{11}$ thigh torsion and $q_{12}$ knee flexion. Parameters: $p_{1}$ arm length, $p_{2}$ torso length, $p_{3}$ half-width of the pelvis, $p_{4}$ pelvis height, $p_{5}$ knee adduction and $p_{6}$ thigh length. [B] Simplified model for the simulation with damped springs $\left(q_{s}\right)$, coupling motion $(\mathrm{f}(q))$ and set coordinates $(k)$.

\section{Simulation Model}

The $12 \mathrm{DoF}$ in the data analysis model were reduced by introducing damped springs, coupling motions and some assumptions for the simulation model (Figure 3B). The displacement of the bar in the sagittal plane $\left(q_{s 1}, q_{s 2}\right)$ and the gymnast's shoulder structure $\left(q_{s 6}\right)$ were modelled as damped linear springs (stiffness $s$ and damping coefficient $c$ ). The model parameters were adjusted using a matching procedure detailed in the next section. An analysis of back flexion $\left(q_{8}\right)$ and thigh torsion $\left(q_{11}\right)$ was carried out and showed relationships with hip flexion and abduction $\left(q_{9-10}\right)$. Back flexion and thigh torsion were then expressed as polynomial functions: $\mathrm{f}_{8}\left(q_{9,}, q_{10}\right)$ and $\mathrm{f}_{11}\left(q_{9,}, q_{10}\right)$. There was also a parameter $\mathrm{f}\left(q_{7}\right)$ that governed the elevation of the scapular girdle as a function of the shoulder flexion angle $\left(q_{7}\right)$ to avoid singularity with $q_{6}$ when the arm and the trunk are aligned, and to obtain a realistic translation of the glenohumeral joint centre while flexing the arm. The assumptions were that the bar only had displacement in the sagittal plane $\left(q_{3}(t)=k_{3}=0\right)$, the gymnast did not translate along the bar $\left(q_{5}(t)=k_{5}=0\right)$ and the knees remained slightly flexed $\left(q_{12}(t)=k_{12}=5^{\circ}\right)$ throughout the movement, as measured in average for the actual movements.

In this seven DoF $\left(q_{s 1, s 2,4, s 6,7,9,10)}\right)$ simulation model, the controlling DoF were the shoulder flexion-extension $\left(q_{7}\right)$, the hip flexion-extension $\left(q_{9}\right)$ and the hip abduction-adduction $\left(q_{10}\right)$ while the bar $\left(q_{s 1}\right.$ and $\left.q_{s 2}\right)$ and the arm lengthening $\left(q_{s 6}\right)$ were modelled as damped springs. Finally the 
arm rotation relative to the bar $\left(q_{4}\right)$ could be solved from the equation of motion which was expressed as an Euler-Lagrange equation:

$\mathbf{M}(\mathbf{q}) \ddot{\mathbf{q}}+\mathbf{N}(\mathbf{q}, \dot{\mathbf{q}})+\mathbf{G}(\mathbf{q})=\boldsymbol{\tau}$,

where $\mathbf{M}(\mathbf{q})$ is the inertia matrix and $\mathbf{q}=\left[q_{1}, \ldots, q_{12}\right]^{\mathrm{T}}$ represents the generalized coordinates. The Coriolis and centrifugal effects are grouped together in $\mathbf{N}(\mathbf{q}, \dot{\mathbf{q}}) ; \mathbf{G}(\mathbf{q})$ is the gravity effect and $\boldsymbol{\tau}$, with $\boldsymbol{\tau}=\boldsymbol{\tau}_{A}+\boldsymbol{\tau}_{P}$, represents the generalized forces comprising the horizontal and vertical forces applied to the bar, the friction between the bar and the hands (considered equal to zero), the force at the arm lengthening spring, and the joint torques generated by the shoulders in flexion/extension and the hip in flexion/extension and abduction/adduction. The variable $\mathbf{q}$ that describes the configuration of the system consists of two different sets: a first set $\mathbf{q} 1$ describing the bar position, the arm rotation and the arm lengthening that are unknown kinematics. A second set $\mathbf{q} 2$ describes the joint angles of the gymnast that are the input of the model. Hence the Lagrangian dynamics (Eq. [1]) becomes:

$\left[\begin{array}{ll}\mathbf{M}_{11} & \mathbf{M}_{12} \\ \mathbf{M}_{21} & \mathbf{M}_{22}\end{array}\right]\left(\begin{array}{c}\ddot{\mathbf{q}} 1 \\ \ddot{\mathbf{q}} 2\end{array}\right)+\left[\begin{array}{l}\mathbf{N}_{1}+\mathbf{G}_{1} \\ \mathbf{N}_{2}+\mathbf{G}_{2}\end{array}\right]=\left[\begin{array}{c}\boldsymbol{\tau} 1 \\ \boldsymbol{\tau} 2\end{array}\right]$

As $\ddot{\mathbf{q}} 2$ is calculated as the second time derivative of quintic functions and $\boldsymbol{\tau} 1$ depends on $\mathbf{q} 1$ and $\dot{\mathbf{q}} 1\left(\boldsymbol{\tau} 1=\left[\begin{array}{llll}-s_{1} q_{s 1}-c_{1} \dot{q}_{s 1} & -s_{2} q_{s 2}-c_{2} \dot{q}_{s 2} & 0 & -s_{6} q_{s 6}-c_{6} \dot{q}_{s 6}\end{array}\right]^{\mathrm{T}}\right), \ddot{\mathbf{q}} 1$ is solved by:

$$
\ddot{\mathbf{q}} 1=\mathbf{M}_{11}^{-1}\left(\boldsymbol{\tau} 1-\mathbf{N}_{1}-\mathbf{G}_{1}-\mathbf{M}_{12} \ddot{\mathbf{q}} 2\right)
$$

Parameters of the simulation model were the segmental inertia parameters, the stiffness $s_{1,2,6}$ and damping $c_{1,2,6}$ coefficients of the bar and shoulder springs. Input to the simulation model comprised the initial state of the bar and the shoulder $\left(q_{1}^{t_{i}}, q_{2}^{t_{i}}, q_{6}^{t_{i}}, \dot{q}_{1}^{t_{i}}, \dot{q}_{2}^{t_{i}}, \dot{q}_{6}^{t_{i}}\right)$, the initial angular position and velocity of the arm $\left(q_{4}^{t_{i}}, \dot{q}_{4}^{t_{i}}\right)$ and the joint angle time histories $\left(q_{7,9,10}^{t_{i} \rightarrow t_{f}}\right)$ expressed as sequences of quintic functions in order to evaluate the first and second time derivatives. Output from the simulation comprised the time histories of the bar displacements and the arm rotation angle. The whole body rotation angle $\varphi$ was calculated as the angle between the upward vertical and the line joining the neutral bar position to the mass centre. Then joint torques, potential and kinetic energies were calculated.

\section{Matching Simulations}

For the model parameters optimization, quintic splines were used to fit the time histories of the recorded generalized coordinates. The simulation model was implemented with a genetic optimization algorithm (Caroll, 1996). A cost function was established to minimise the root mean square difference (tilde notation represents the square difference) between the recorded and simulated performances described by the bar displacement $q_{1-2}$, the arm rotation $q_{4}$ and arm lengthening $q_{6}$. The kinematics of the five stalders were considered together:

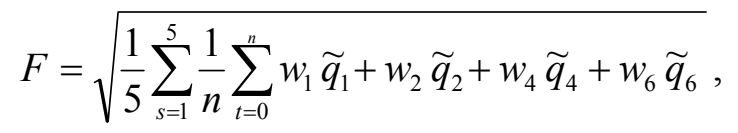

The weightings, $w_{i}$, were chosen so that each component of the cost function made approximately equal contributions based on the analysis of the actual performances. The following parameters were allowed to vary in order to improve the match between the recorded and 
simulated performances. The stiffness and damping coefficients of the bar were allowed to vary between 20,000 and 27,500 Nm $\mathrm{Nm}^{-1}$ and between 0 and $500 \mathrm{Nsm}^{-1}$ to conform to the specifications of the FIG (2006). The stiffness and damping coefficients of the arm lengthening spring were allowed to vary over wider ranges $\left(10,000-20,000 \mathrm{Nm}^{-1}\right.$ and $\left.500-2000 \mathrm{Nsm}^{-1}\right)$ than those of the bar springs. In line with the accuracy of the model of Yeadon (1990), the segmental masses (for segments 1-2 \& 5-7, referred to Figure 3) and moments of inertia (for segments $2 \& 4-7$ ) were allowed to vary between $\pm 8 \%$ and $\pm 10 \%$ (1\% error in measurement for each dimension to calculate the volume and $5 \%$ error in density estimation) respectively, while the total body mass was constrained to remain at its measured value. These bounds were not reached in the matching optimization.

\section{Optimization}

The stalder was modelled with series of five polynomial functions for the shoulder flexion/extension, four for hip flexion/extension and five for the hip abduction/adduction, that were characterized by their duration $\left(\mathbf{D}_{i}\right)$ and final angle magnitude $\left(\mathbf{Q}_{i}\right)$. These numbers were sufficient to match the recorded kinematics with a maximum RMS error of $5^{\circ}$ (for a total range of motion of $\left.110^{\circ}\right), 4^{\circ}\left(165^{\circ}\right)$ and $3^{\circ}\left(70^{\circ}\right)$ for the shoulder flexion, hip flexion and abduction, respectively. During each part (except for the first part for the hip abduction) the joint angles $\left(q_{7}\right.$, $\left.q_{9}, q_{10}\right)$ were allowed to change independently. In changing a joint angle $q$ from $q_{i}$ to $q_{j}$ between times $t_{i}$ and $t_{j}$, the time history was given by a quintic function that fits well gymnast kinematics on high bar (Yeadon \& Hiley, 2000): $q(x)=q_{i}+\left(q_{j}-q_{i}\right) u(x)$ where $u(x)=x^{3}\left(6 x^{2}-15 x+10\right)$ with $x=\left(t-t_{i}\right) /\left(t_{j}-t_{i}\right)$ so that $\dot{q}=\ddot{q}=0$ at $t_{i}$ and $t_{f}$. A genetic algorithm (Caroll, 1996) was used to manipulate the phase durations and the extreme joint angles of the shoulder and the hip in order to minimize the cost function, the integral quadratic amount of active torques throughout the motion:

$J=\int_{t_{i}}^{t_{f}} \frac{1}{2} \boldsymbol{\tau}_{A}(t)^{\mathrm{T}} \mathbf{W} \boldsymbol{\tau}_{A}(t) d t$,

where $\mathbf{W}$ is a weighting matrix defined from actual performances and $\boldsymbol{\tau}_{A}$ the active generalised forces. The cost function is subject to the system dynamics $\dot{\mathbf{x}}=f(t, \mathbf{x}, \boldsymbol{\tau})$, where $\mathbf{x}=[\mathbf{q}, \dot{\mathbf{q}}]^{\mathrm{T}}$ is the state vector of the system, under constraints:

$$
\left\{\begin{array}{c}
\theta_{i}\left(t_{i}, \mathbf{x}_{i}\right)=0 \\
\theta_{f}\left(t_{f}, \mathbf{x}_{f}\right) \leq 0 \\
h(t, \mathbf{x}) \leq 0 \\
g\left(t, \mathbf{x}, \boldsymbol{\tau}_{A}\right) \leq 0 .
\end{array}\right.
$$

The initial state was prescribed $\left(\theta_{i}\right)$ and at the final rotation angle, greater than $365^{\circ}$, the hip and shoulder angles had to be less than $5^{\circ}\left(\theta_{f}\right.$ final state). These values were chosen to lie within the FIG (2006) rules. An additional path constraint $h(t, \mathbf{x})$ was imposed to obtain a piked straddled posture (i.e. legs apart and lower than the bar in the arms local frame) under the bar and to avoid any body collision (lower limbs relative to the trunk, upper limbs or bar). The control constraint $g(t, \mathbf{x}, \tau)$ was used to ensure that the generalized forces did not exceed the gymnast's strength.

\section{Active and passive torques}

The joint torque at the shoulder and the hip was the sum of the passive and active torques. Since the knee remained slightly flexed, a double exponential model (Hoang et al., 2005; Silder et al., 2007) defined the passive torque such that the torque magnitude was equal to an arbitrary value of 
$100 \mathrm{Nm}$ at a desired end-range of motion (100 Nm of flexor torque at maximal extension $q^{\text {min }}$, and $100 \mathrm{Nm}$ of extensor torque at maximum flexion $\left.q^{\max }\right)$ :

$$
\left\{\begin{array}{c}
\tau_{P}(q)=B_{1} \exp ^{k_{1} q}+B_{2} \exp ^{k_{2} q} \\
\tau_{P}\left(q^{\min }\right)=100 \mathrm{Nm} \\
\tau_{P}\left(q^{\max }\right)=-100 \mathrm{Nm} .
\end{array}\right.
$$

The arbitrary value was chosen after measurements on an isokinetic dynamometer. This function represents the net passive torque due to muscle stretching and other structures (e.g. soft connective tissue loads and ligament forces). Due to gravitational and centrifugal effects, the passive hip extensor torque could exceed $-100 \mathrm{Nm}$ slightly and the hip flexion angle could exceed the hip flexion limit by a few degrees. The maximum active torque was defined as a function of joint angle and angular velocity: $\boldsymbol{\tau}_{A}^{\max }=f(q, \dot{q})$ for the shoulder and the hip (Yeadon et al., 2006; King et al., 2006). Since it was not possible to collect data on isokinetic ergometer with this gymnast, the model of another gymnast was scaled in order to respect the joint torques calculated using the stalder experimental. The kinematics was optimized for four hip flexion flexibilities $q_{9}^{\max }: 100^{\circ}, 110^{\circ}, 120^{\circ}$ and $130^{\circ}$. From times histories and key values of kinematics (time at mass centre-bar angle $\varphi=\left\{90^{\circ}, 180^{\circ}, 270^{\circ}, 360^{\circ}\right\}$, straddled pike period, maximum hip flexion and abduction), kinetics (shoulder and hip torque under the bar) and energy (potential, kinetic $E_{k}=\frac{1}{2} \mathbf{q}^{T} \mathbf{M}(\mathbf{q}) \mathbf{q}$ - and total), the changes in technique with a decrease in flexibility were assessed.

\section{Results}

For five recorded stalders (Eq.[4]), the stiffness coefficients of the bar obtained in the matching procedure were 20,024 and $20,007 \mathrm{Nm}^{-1}$ for the horizontal and vertical bar displacements respectively, which lay within the limits as set out by the FIG (2006). The damping coefficients were also close, 499 versus $498 \mathrm{Nsm}^{-1}$. The parameters for the arm lengthening $\left(q_{s 6}\right)$ had a stiffness of $12,000 \mathrm{Nm}^{-1}$ and a damping coefficient of $1,473 \mathrm{Nsm}^{-1}$.

For each of the four values of hip flexibility, an optimum movement which minimized the cost function (Eq. [5]) while respecting the constraints (Eq. [6]) was found; the stalders were performed with a straddled piked posture under the bar to reach a nominal handstand without body collisions (Figure 4). As the flexibility decreased, the scores $J$ in Eq. [5] (and hence the active joint torques) increased. In general, the kinematics and the kinetics of the four flexibility conditions were similar while the energy time history of the $100^{\circ}$ flexibility condition differed from the other conditions. In the actual stalders (Figure 2) compared to all of the optimized movements, the gymnast attained the piked posture later, began the shoulder opening earlier and had greater hip flexion under the bar. 


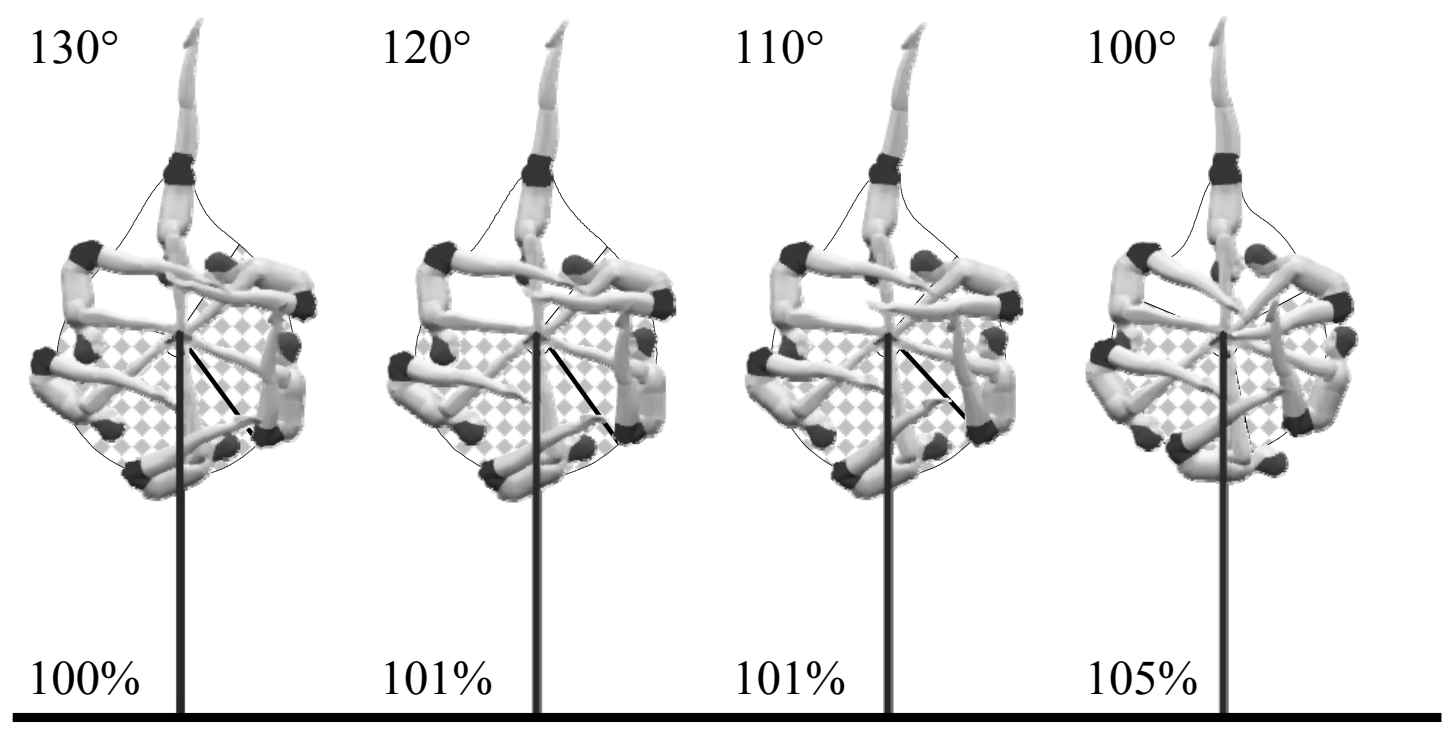

Figure 4: Optimum performances of stalder for four hip flexibilities: $130^{\circ}, 120^{\circ}, 110^{\circ}$ and $100^{\circ}$.

Note: the path of the centre of mass (dashed line) and the sector where the gymnast is in a piked posture (filled with solid diamond) with the maximum hip flexion (bold line) give additional information about the kinematics.

Table 1: Key values for the optimum stalder for four hip flexibilities

\begin{tabular}{|c|c|c|c|c|}
\hline Stalder & $130^{\circ}$ & $120^{\circ}$ & $110^{\circ}$ & $100^{\circ}$ \\
\hline Score & $100 \%$ & $101 \%$ & $101 \%$ & $105 \%$ \\
\hline piked & $34-285^{\circ}$ & $38-283^{\circ}$ & $41-282^{\circ}$ & $62-293^{\circ}$ \\
\hline $\begin{array}{l}\text { posture } \\
\text { [duration] }\end{array}$ & {$[0.93 \mathrm{~s}]$} & {$[0.89 \mathrm{~s}]$} & {$[0.86 \mathrm{~s}]$} & {$[0.73 \mathrm{~s}]$} \\
\hline time at & $1.32 \mathrm{~s}$ & $1.32 \mathrm{~s}$ & $1.34 \mathrm{~s}$ & $1.28 \mathrm{~s}$ \\
\hline$\varphi=90^{\circ}$ & $1.61 \mathrm{~s}$ & $1.61 \mathrm{~s}$ & $1.63 \mathrm{~s}$ & $1.53 \mathrm{~s}$ \\
\hline$\varphi=180^{\circ}$ & $1.94 \mathrm{~s}$ & $1.94 \mathrm{~s}$ & $1.95 \mathrm{~s}$ & $1.82 \mathrm{~s}$ \\
\hline $\begin{array}{l}\varphi=270^{\circ} \\
\varphi=360^{\circ}\end{array}$ & $2.60 \mathrm{~s}$ & $2.57 \mathrm{~s}$ & $2.54 \mathrm{~s}$ & $2.60 \mathrm{~s}$ \\
\hline $\max q_{9}$ & $\begin{array}{l}121^{\circ} \\
\left(\varphi=143^{\circ}\right)\end{array}$ & $\begin{array}{l}118^{\circ} \\
\left(\varphi=144^{\circ}\right)\end{array}$ & $\begin{array}{l}112^{\circ} \\
\left(\varphi=137^{\circ}\right)\end{array}$ & $\begin{array}{l}99^{\circ} \\
\left(\varphi=167^{\circ}\right)\end{array}$ \\
\hline $\max q_{10}$ & $29^{\circ}$ & $36^{\circ}$ & $36^{\circ}$ & $40^{\circ}$ \\
\hline at $\varphi=180^{\circ}$ & $\begin{array}{l}\tau_{2}=2422 \mathrm{~N} \\
q_{7}=70^{\circ}, \\
\tau_{7}=274 \mathrm{Nm} \\
q_{9}=121^{\circ}, \\
\tau_{9}=-60 \mathrm{Nm}\end{array}$ & $\begin{array}{l}\tau_{2}=2443 \mathrm{~N} \\
q_{7}=70^{\circ}, \\
\tau_{7}=287 \mathrm{Nm} \\
q_{9}=118^{\circ}, \\
\tau_{9}=-16 \mathrm{Nm}\end{array}$ & $\begin{array}{l}\tau_{2}=2414 \mathrm{~N} \\
q_{7}=71^{\circ}, \\
\tau_{7}=311 \mathrm{Nm} \\
q_{9}=111^{\circ}, \\
\tau_{9}=35 \mathrm{Nm}\end{array}$ & $\begin{array}{l}\tau_{2}=2634 \mathrm{~N} \\
q_{7}=97^{\circ}, \\
\tau_{7}=317 \mathrm{Nm} \\
q_{9}=99^{\circ}, \\
\tau_{9}=-18 \mathrm{Nm}\end{array}$ \\
\hline
\end{tabular}

The total duration of the stalders ranged from 2.54 to $2.60 \mathrm{~s}$. The timing (time at $90^{\circ}, 180^{\circ}$, $270^{\circ}$ and $360^{\circ}$ of rotation) was not really affected by gymnast flexibility; only at $100^{\circ}$ the gymnast was slightly faster from $\varphi=0^{\circ}$ to $180^{\circ}$ and slower in the fourth quadrant. With decreasing flexibility, the piked posture started later $\left(34^{\circ}\right.$ to $\left.62^{\circ}\right)$ and its duration diminished (Table 1). In all the flexibility conditions the pike was maintained at least until $280^{\circ}\left(282-293^{\circ}\right)$. Under the bar, the postures differed slightly, the shoulder angle and hip abduction angle increased with decreased hip 
flexion. These changes in posture minimize the effects of the centrifugal force and gravity on the active joint torques by the contribution of the hip passive torque. For the four optimized stalders, the maximum value of the hip flexion $\left(121^{\circ}, 118^{\circ}, 112^{\circ}, 99^{\circ}\right.$ for $130^{\circ}, 120^{\circ}, 110^{\circ}$ and $100^{\circ}$ flexibilities) was close to the value which corresponded to a passive torque of $-100 \mathrm{Nm}$ and only exceeded this for the $110^{\circ}$ flexibility performance.

The kinetics was also affected by the hip flexibility (Figure 5). The vertical force applied to the bar exceeded $2400 \mathrm{~N}$ for $110-130^{\circ}$ flexibility and $2600 \mathrm{~N}$ at $100^{\circ}$ flexibility, i.e. more than four body weights. In general, the gymnast produced an eccentric shoulder flexor torque from $\varphi=0^{\circ}$ to $80^{\circ}$ followed by an extensor torque until $280^{\circ}$ and then a flexor torque again to reach handstand. The maximum shoulder extensor torque value always occurred under the bar and increased with decreased flexibility. The maximum shoulder flexor torque values (at the beginning and before handstand) also showed an upward trend from $130^{\circ}$ to $100^{\circ}$ hip flexibilities.

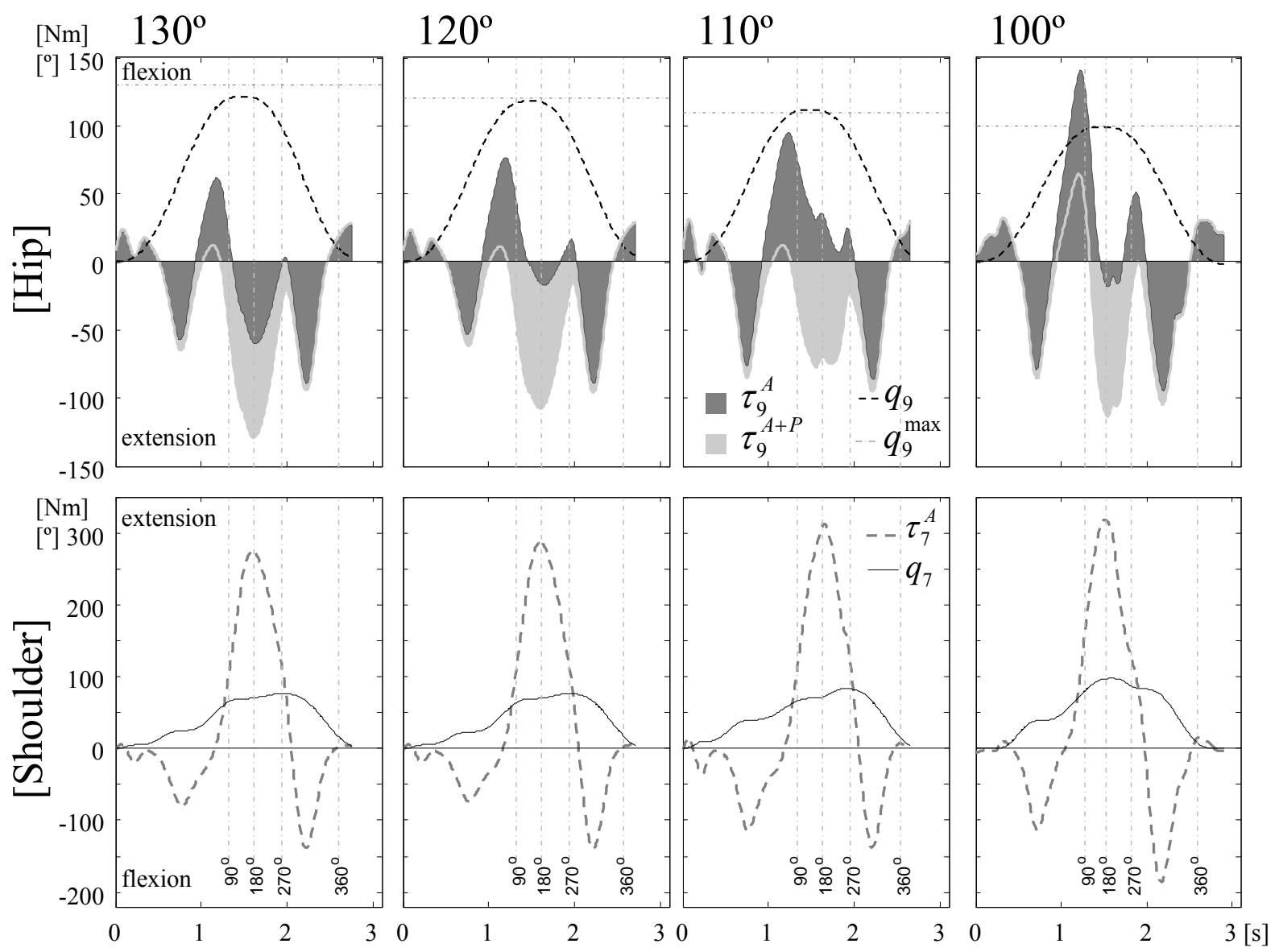

Figure 5: Time histories of the kinematics $\left(q_{9}\right)$ and kinetics (total torque $\tau_{9}^{A+B}$ and active torque $\tau_{9}^{A}$ ) of the hip flexion-extension. Time histories of the shoulder angle $\left(q_{7}\right)$ and active torque $\left(\tau_{7}\right)$.

Note: Vertical dashed lines represent the body rotation $\varphi$ at $90^{\circ}, 180^{\circ}, 270^{\circ}$ and $360^{\circ}$.

A peak hip flexor torque generally appeared just before $90^{\circ}$ of body rotation and a peak hip extensor torque before reaching handstand. However the magnitude of the first peak and the 
kinetics from $\varphi=90^{\circ}$ to $270^{\circ}$ changed markedly with flexibility. As with shoulder torque, peaks of hip flexion joint torque increased with decreased flexibility $\left(62,77,95\right.$ and $141 \mathrm{Nm}$, from $130^{\circ}$ to $100^{\circ}$ flexibility). In the $130^{\circ}$ flexibility condition the average joint torque while the gymnast was below the horizontal $\left(90^{\circ}<\varphi<270^{\circ}\right)$ was $-34 \mathrm{Nm}$ (mainly hip extensor torque); this value became zero in the $120^{\circ}$ flexibility condition and then positive $(31$ and $20 \mathrm{Nm})$ in $110^{\circ}$ and $100^{\circ}$ conditions. In the four cases, the total energy (Figure 6) decreased sharply at the beginning of the movement, stayed nearly constant until $\varphi=90^{\circ}$ before decreasing again until $180^{\circ}$. After the body passed through the lower part of the circle, the energy increased slowly and then faster $\left(\varphi>330^{\circ}\right)$, especially for $100^{\circ}$ hip flexibility. The potential and kinetic energies from $110^{\circ}$ to $130^{\circ}$ flexibilities were similar while the change in energy for the $100^{\circ}$ flexibility was smaller mainly due to the potential energy.

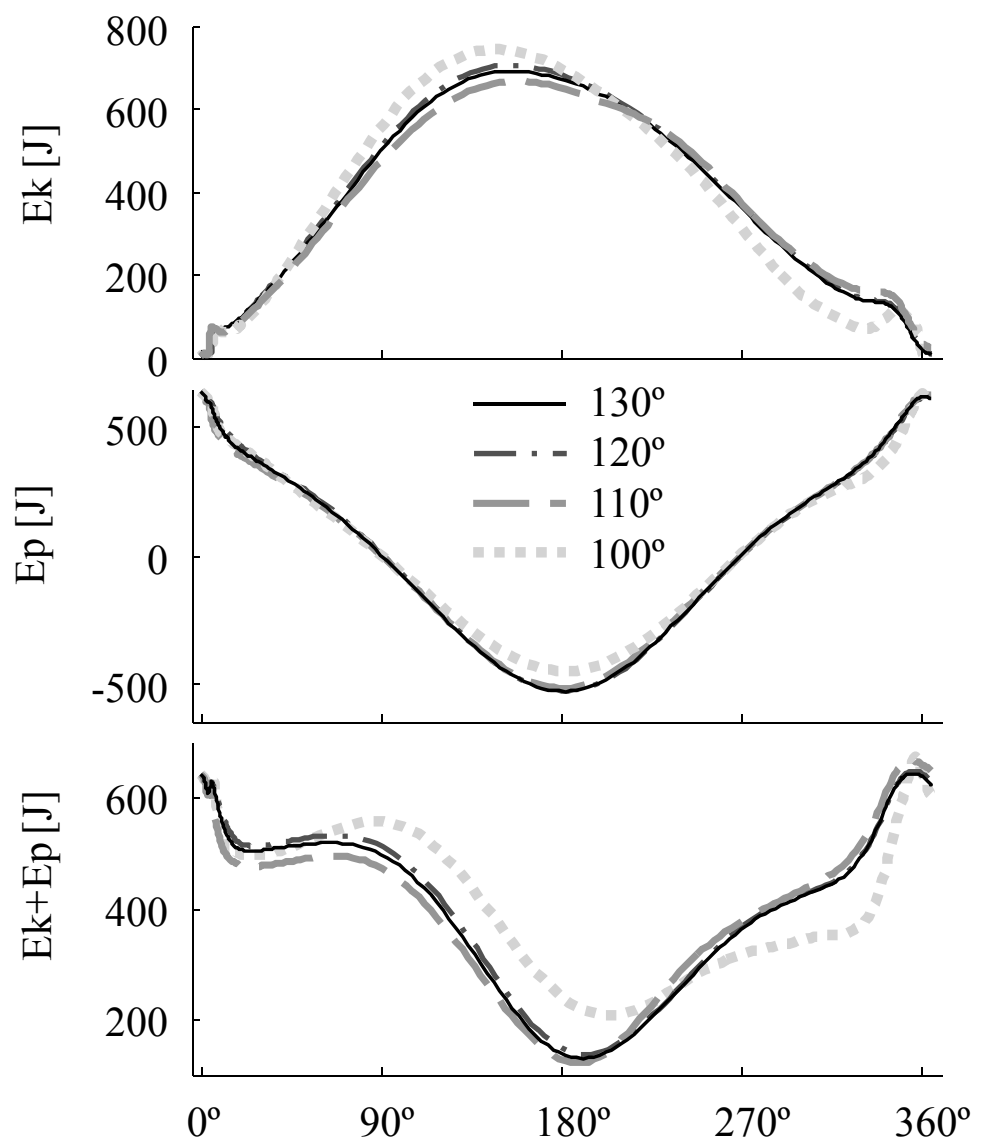

Figure 6: Kinetic (Ek), potential (Ep) and total (Ek+Ep) energies for the four as functions of the body rotation $\varphi$.

\section{Discussion}

This study has shown that for a wide range of hip flexibility $\left(100-130^{\circ}\right)$ gymnasts can perform a straddled stalder from handstand to handstand with a decreased least torque norm by modifying the coordination. A flexible gymnast minimizes the peaks of hip and shoulder torques by piking earlier and having greater shoulder flexion. The early piking decreases the potential energy and results in a smaller centrifugal force under the bar. For a stiff gymnast, the centrifugal forces allow the piked 
posture to be maintained under the bar, moreover the hip abduction increased. In all the flexibility conditions, the performance is partially a balance between gravitational and centrifugal effects and the torque generated by the passive structures of the musculoskeletal system.

Scaling the maximum joint torques using the dynamics of actual stalders was an adequate method in regard to the chosen cost function. In order to optimize the performance of an elite gymnast a subject-specific data collection on an isokinetic ergometer would be necessary to obtain realistic results. The previous gymnast models for high bar movements (Hiley and Yeadon, 20032007) have been adapted to the stalder specific dynamics by introducing a passive torque model, an extra DoF between the torso and the pelvis and by coupling motions (personalised behaviour of the scapular girdle elevation as a function of arm flexion as well as back flexion and hip torsion as functions of hip flexion and abduction which allowed realistic kinematics without increasing DoF. Since the purpose was to compare optimum stalders for different hip flexibilities, the arbitrary value of $100 \mathrm{Nm}$ was not a limit of our study. However for other purposes - performance optimization and analysis of mechanical work (Silder et al., 2008) or muscular force estimation for actual performances (Vigouroux et al., 2006) -, a subject-specific model would be important. The passive torque model could be improved by considering both hip flexion and abduction using an isokinetic dynamometer and electromyography.

The hip active torque under the bar is small (e.g. at $120^{\circ}$ flexibility condition) and so the majority of the torque is passive. This result is consistent with practice in that gymnasts do not feel they produce large hip torques to maintain the pike. From a coaching point of view, the most difficult to learn is the control of the angular velocity in the first part and then the balance under the bar to avoid an early body opening. However the accuracy of the model is difficult to experimentally assess because an active torque due to stretching reflex is added to the passive torque. This simulation model will be appropriate for studying and optimizing other gymnastics skills with straddled legs (e.g. endo and lift to handstand) or with large hip and back flexion (e.g. dislocation on high bar and swings on parallel bars for double piked somersault dismounts).

The stalders were obtained using a global genetic optimization in a large solution space. The discretization of the parameters (128 bit binary encoding, i.e. a minimum resolution of $0.008 \mathrm{~s}$ and $1^{\circ}$ ) was sufficient in line with the work of Hiley and Yeadon (2007) on the coordination robustness on high bar. For a flexibility of $130^{\circ}-120^{\circ}$, the algorithm was rapid in finding a solution which respected the constraints while the space of possible solutions became smaller for a stiffer gymnast. The algorithm did not find a feasible solution for a flexibility less than $100^{\circ}$ because the torque, required to attain the piked posture, exceeded the gymnast's strength or there were collisions of the legs with the bar. An approach based on the coordination robustness (Hiley and Yeadon, 2007) by introducing error in the time of the "closing phase" could bring additional information on the stiff gymnast's ability to perform the stalder consistently.

While only three DoF were controlled, the comparison of the four flexibility conditions remains difficult because the movement is subject to important centrifugal and gravitational effects which can compensate for each other or can be compensated for by the passive torque. Hence, there may exist several local minima which make the global optimization difficult. In general, from $110^{\circ}$ to $130^{\circ}$, the change in flexibility does not penalize the gymnast and small compensations occur. For a $100^{\circ}$ hip flexion flexibility, the coordination is more affected. To To simplify the discussion of results, movement can be divided into three phases: [1] from handstand to the piked posture, [2] swing in a piked posture and [3] opening phase to complete stalder to handstand. As we have chosen a least squared force criterion, the mechanical aim of each phase is [1] to minimize the energy of the system (decrease the potential energy to minimize the joint torques 
under the bar but also to reach handstand), [2] to optimize the piked posture under the bar (balance between inertial and gravitational effects and passive hip torque in relation to gymnast's flexibility) and [3] to control the lift to handstand with a small amount of kinetic energy.

During the first phase of the stalder, the total energy decreases with a slow and large hip flexion and a small shoulder extension. This movement requires eccentric contraction for both hip and shoulder joints. The beginning of the piked posture occurs progressively later with decreased flexibility but the mechanical energies are similar at this instant for the four flexibility conditions. In competition, the gymnast can either use this technique or begin the stalder with a large shoulder extension to be able to increase the kinetic energy with a large and fast hip flexion. In general for elite gymnasts, the piked posture begins later - the centre of gravity remaining far from the bar (Figure 2) - in order to produce a greater amount of energy. Hence gymnasts have a larger margin of error in the third phase of the movement to complete the movement without deviation or to perform longitudinal turns before handstand. This strategy explains the main differences between actual and simulated performances. Since the angular velocity under the bar is greater in actual performances, the passive structures are more extended and gymnasts reach a greater hip flexion.

In the second phase, the shoulder angle and hip abduction angle compensate for the hip flexion. These angle adjustments are small (less than $10^{\circ}$ ) between $110^{\circ}$ and $130^{\circ}$ of hip flexibility but have consequences for the hip kinetics. In the $120^{\circ}$ flexibility condition, the piked posture requires only low active torques (on average equal to zero) because the passive torque compensates for gravitational and inertial effects. In contrast to this balanced condition, the $110^{\circ}$ flexible gymnast has to generate a hip flexor torque to maintain the posture and the $130^{\circ}$ flexible gymnast an extensor torque to avoid an over-inverted posture. Greater hip flexion for reducing the active hip extensor torque would require more shoulder flexion but the shoulder is weak at this angle in relation to the strength model. This kind of coordination is nevertheless possible for female gymnasts who are very flexible at the hips but cannot produce large shoulder torques. In this case, stalders are performed with the arms aligned with the trunk and a hip plus back flexion higher than $180^{\circ}$ that would cause smaller shoulder torques. Future optimizations using a joint torque model specific to female gymnast should support this statement.

In the $100^{\circ}$ flexibility condition, the moment of inertia about the bar is smaller and creates a faster rotation and a higher kinetic energy. In the third phase, large shoulder and hip torques (opening actions) are required to reach handstand since the angular velocity is small, the fourth quadrant is made in 0.6 to $0.7 \mathrm{~s}$. The centre of mass paths (Figure 4) show that this third phase is mainly a vertical displacement and requires balance control. The optimized solutions minimize the integral quadratic amount of active torques and differ from the actual movements of the elite gymnast. However the optimized simulations are similar to the techniques used by novice gymnasts when learning stalder. In the performances by the elite gymnast (Figure $2 \mathrm{~A}, \mathrm{~B}, \mathrm{E}$ ) the arms are aligned with the trunk in the fourth quadrant, unlike the optimized simulations. This may be because it facilitates the performance of longitudinal turns. Thus unlike the novice gymnasts who may indeed minimize joint torque, there are other considerations which influence the technique used by elite gymnasts. Future simulations will be carried out to maximize angular momentum or minimize joint torque in the fourth quadrant of rotation to find the stalder technique which would be the most suitable for gymnast progression.

To summarise, the hip flexion flexibility has an effect on the dynamics of straddled stalder from handstand to handstand. From $110^{\circ}$ to $130^{\circ}$ hip flexibly, the kinematics are similar and the peak shoulder and hip torques increase with decreased flexibility. For a $100^{\circ}$ hip flexibility, there 
are larger compensations between the hip and shoulder angles. The movement requires higher torques to reach the piked posture and to complete the stalder to handstand. Greater flexibility results in less physical demand on the gymnast.

\section{References}

Arampatzis A, Brüggemann GP. 2001. Mechanical energetic processes during the giant swing before the Tkatchev exercise. J Biomech. 34:505-512.

Arampatzis A, Brüggemann GP. 1998. A mathematical high bar-human body model for analysing and interpreting mechanical-energetic processes on the high bar. J Biomech. 31:1083-1092.

Begon M, Wieber P, Yeadon MR. 2008. Kinematics estimation of straddled movements on high bar from a limited number of skin markers using a chain model. J Biomech. 41:581-586.

Carroll DL. 1996. Chemical Laser Modeling with Genetic Algorithms. AIAA Journal. 34:338-346.

Davy DT, Audu ML. 1987. A dynamic optimization technique for predicting muscle forces in the swing phase of gait. J Biomech. 20:187-201.

Ehrig RM, Taylor WR, Duda GN, Heller MO. 2006. A survey of formal methods for determining the centre of rotation of ball joints. J Biomech. 39:2798-2809.

FIG. 2006. Fédération Internationale de Gymnastique. Manual apparatus norms. Part IV testing procedures. FIG, Switzerland.

Hiley MJ, Yeadon MR. 2003a. Optimum technique for generating angular momentum in accelerated backward giant circles prior to a dismount. J Appl Biomech. 19:119-130.

Hiley MJ, Yeadon MR. 2003b. The margin for error when releasing the high bar for dismounts. J Biomech. 36:313-319.

Hiley MJ, Yeadon MR. 2005a. Maximal dismounts from high bar. J Biomech. 38:2221-2227.

Hiley MJ, Yeadon MR. 2005b. The margin for error when releasing the asymmetric bars for dismounts. J Appl Biomech. 21:223-235.

Hiley MJ, Yeadon MR. 2007. Optimization of backward giant circle Technique on the asymmetric bars. J Appl Biomech. 23:300-308.

Hiley MJ, Yeadon MR, Buxton E. 2007. Consistency of performances in the Tkatchev release and re-grasp on high bar. Sports Biomech. 6:121-30.

Hoang PD, Gorman RB, Todd G, Gandevia SC, Herbert RD. 2005. A new method for measuring passive length-tension properties of human gastrocnemius muscle in vivo. J Biomech. 38:1333-1341.

King MA, Wilson C, Yeadon MR. 2006. Evaluation of a torque driven model of jumping for height. J Appl Biomech. 22:264-274.

Predescu G. 1985. Bazele mecanice ale lucrului la bara fixă în gymnastica masculină. Educatie Fizica Si Sport 9:27-32.

Silder A, Whittington B, Heiderscheit B, Thelen DG. 2007. Identification of passive elastic joint moment-angle relationships in the lower extremity. J Biomech. 40:2629-2635.

Silder A, Heiderscheit B, Thelen DG. 2008. Active and passive contributions to joint kinetics during walking in older adults. J Biomech. 41:1520-1527.

Vigouroux L, Quaine F, Labarre-Vila A, Moutet F. 2006. Estimation of finger muscle tendon tensions and pulley forces during specific sport climbing grip techniques. J Biomech. 39:2583-2592.

Wieber P, Billet F, Boissieux L, Pissard-Gibollet R. 2006. The HuMAnS toolbox, a homogeneous framework for motion capture, analysis and simulation. Ninth International Symposium on the $3 \mathrm{D}$ analysis of human movement. 
Yeadon MR. 1990. The simulation of aerial movement - II. A mathematical inertia model of the human body. J Biomech. 23:67-74.

Yeadon MR, Hiley MJ. 2000. The mechanics of the backward giant circle on the high bar. Hum Movement Sci. 19:153-173.

Yeadon MR, King MA, Wilson C. 2006. Modelling the maximum voluntary joint torque/angular velocity relationship in human movement. J Biomech. 39:476-482.

Yoon YS, Mansour JM. 1982. The passive elastic moment at the hip. J Biomech. 15:905-910. 